\title{
FORCED CONVECTION FROM AN ISOTHERMAL CIRCULAR CYLINDER PLACED IN A CROSS STREAM OF MICROPOLAR FLUID
}

\author{
F. M. Mahfouz \\ Mechanical Power Department, Faculty of Engineering, \\ Menoufiya University, Shebin El-kom, \\ Egypt
}

\begin{abstract}
This paper investigates forced convection associated with the cross-flow of micropolar fluids over a horizontal heated circular cylinder. The full conservation equations of mass, linear momentum, angular momentum and energy are solved to give the details of flow and thermal fields. Heat convection process are mainly influenced by Reynolds number, Prandt/ number and material parameters of micropolar fluid. The Reynolds number is considered up to 200 while the Prandtl number is fixed at 0.7 . The vortex viscosity is the only material parameter considered in this study and is selected in the range from 0 to 5 . The study has shown that generally the mean heat transfer decreases as the vortex viscosity increases. The results have also shown that both the natural frequency of vortex shedding and the amplitude of oscillating lift force experience clear reduction as the vortex viscosity increases.

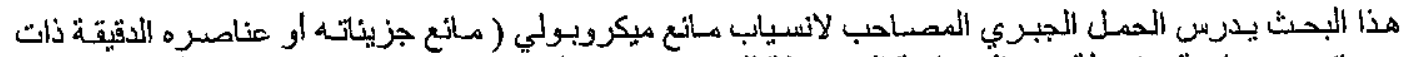

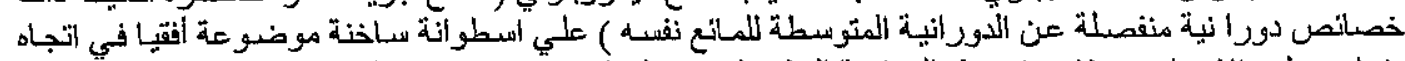

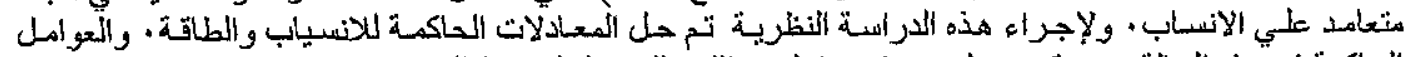

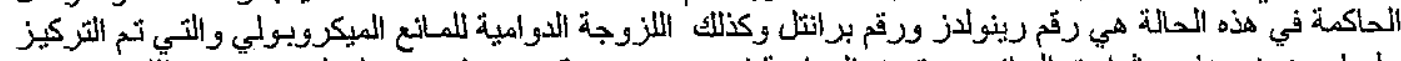

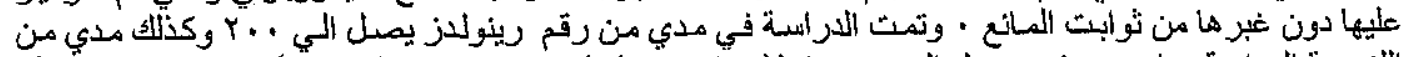

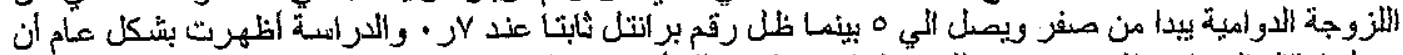

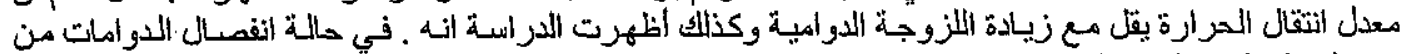

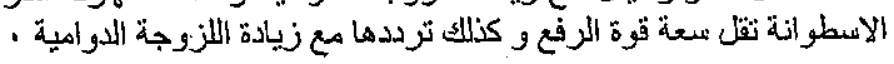

Keywords:- Micropolar fluid-cylinder-forced convection-vortex viscosity-vortex shedding

\section{INTRODUCTION}

The problem of heat convection and hydrodynamics associated with fluid flow over a circular cylinder has received great attention both theoretically and experimentally $[1-6]$. This is not only due to fundamental aspects of the problem but also due to its importance in many practical engineering applications. These applications include nuclear reactors, heat exchangers, hot wires, steam pipes and off-shore structures. Moreover, the interaction between círcular cylinder and its surrounding viscous stream is a good model problem for studying heat and fluid flow over bluff bodies.

Many industrial equipment deal with certain nonNewtonian fluids, such as ferro liquids colloidal fluids, heterogeneous mixtures, exotic lubricants, most slurries and some liquids with polymer additives. For the best design of these equipment, the extension of the studies to explore and understand the behavior of such fluids is important. One of the theories which is expected to describe successfully the behavior of these fluids is the theory of micropolar fluids. Micropolar fluid theory formulated by Eringen [7] takes into account the local effects arising from microstructure and intrinsic motions of the fluid elements. Micropolar fluids can support surface and body couples which are not present in the theory of Newtonian fluids The extension of micropolar fluids theory to deal with thermomicropolar fluids has been given by Eringen [8].

The volume of previous studies of both flow and heat convection problems related to micropolar is relatively small. Arimen et al. [9] has thoroughly reviewed the literature up to 1973 . In the last two decades there was an escalated interest in the theoretical studies related to heat convection in micropolar fluids. These studies however, mostly 
focused on heat convection through boundary layer flow over flat plates and vertical cylinders. Gorla [10] investigated the heat transfer characteristics of a micropolar boundary layer in a cross flow over nonisothermal circular cylinder. Hassanien et al. [11] investigated combined convection on a vertical slender cylinder. The axisymmetric thermal boundary layer of a micropolar fluid on a cylinder was solved by Gorla [12] while the mixed convection in an axisymmetric stagnation flow of micropolar fluid on a vertical cylinder was studied by Mohammedien et al. [13]. Hassanien and Salama [14] studied the flow and heat transfer of a micropolar fluid in an axisymmetric stagnation flow on a cylinder while Gorla and Takhar [15] studied the unsteady mixed convection boundary layer flow of a micropolar fluid near the lower stagnation point on a cylinder. In a recent study Mansour et al. [16] studied heat and mass transfer in magnetohydrodynamic flow of micropolar fluid on a circular cylinder with uniform heat and mass flux.

The aforementioned studies are mostly based on the solutions of boundary layer equations. Such solutions are invalid in the regions of flow separation as that resulting in the cylinder wake. Needless to say that these solutions can not trace the vortex shedding from the cylinder surface and can not predict the resulting oscillating heat transfer and hydrodynamics forces. It is well known that as the flow Reynolds number exceeds a certain value (about 40 in Newtonian fluids) the boundary layer separates alternately and periodically from the upper and lower sides of the cylinder, forming the well known Karman vortex street. The process of vortex shedding causes unsteady flow behavior close to the cylinder which in turn results in oscillating lift and drag forces acting on the cylinder. In response to these oscillating forces the cylinder may vibrate. The cylinder vibrations resulting from the shed vortices can cause structural fatigue and, in certain circumstances, can lead to drastic failure of the structure.

The lack of the information about the effect of vortex shedding on both hydrodynamics and heat transfer associated with micropolar fluid flow over cylinders was the motivation for this study. To the author's knowledge, this is the first study focuses on the vortex shedding process in case of micropolar fluids. The study considers the effect of Reynolds number and material parameters on vortex shedding, hydrodynamics forces and forced convection associated with the cross flow of micropolar fluids over horizontal circular cylinder. In order to predict the vortex shedding process the full governing equations in its elliptic form are considered and solved using a highly accurate numerical technique.

\section{NOMENCLATURE}

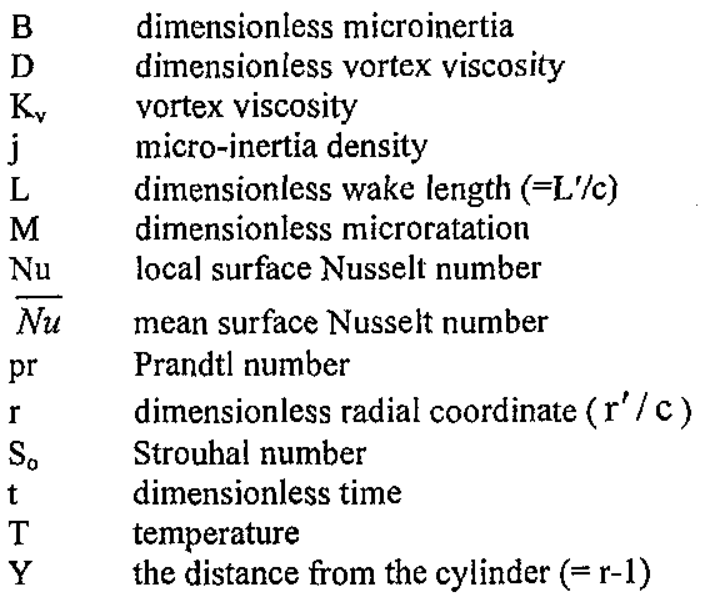

\section{Greek symbols}

$\alpha \quad$ thermal diffusivity

$\phi \quad$ dimensionless temperature

$\gamma \quad$ spin gradient viscosity

$\mu \quad$ viscosity coefficient.

$\lambda$ dimensionless spin gradient viscosity

$\sigma \quad$ microrotation vector in $r^{\prime}, \theta$ plane

\section{Subscripts \\ s at cylinder surface \\ $\infty$ very faraway from the cylinder surface}

\section{PROBLEM FORMULATION}

In the problem considered, a circular isothermal cylinder of radius $\mathrm{c}$ is placed horizontally in a uniform cross-stream of micropolar fluid of temperature $T_{\infty}$. The cylinder surface temperature is kept constant at $\mathrm{T}_{s}\left(\mathrm{~T}_{s}>\mathrm{T}_{\infty}\right)$. The micropolar fluid flow is assumed two-dimensional and the viscous dissipation and microrotation heat conduction are assumed negligible. In polar coordinates $\left(r^{r}, \theta\right)$, where $\theta$ is measured counterclockwise from the rearmost point on the cylinder surface and under the above assumptions the conservation equations of motion and energy in terms of the vorticity, stream function, microrotation and temperature can be expressed as

$$
\begin{aligned}
& \frac{\partial \zeta^{\prime}}{\partial \tau}=\left(\frac{\mu+K_{v}}{\rho}\right) \nabla^{2} \zeta^{\prime}+\frac{1}{r^{\prime}} \frac{\left[\psi, \zeta^{\prime}\right]}{\left[\mathrm{r}^{\prime}, \theta\right]}-\frac{\mathrm{K}_{v}}{\rho} \nabla^{2} \sigma \\
& \zeta^{\prime}=-\nabla^{2} \psi^{\prime} \\
& \frac{\partial \sigma}{\partial \tau}=\frac{\gamma}{\rho \mathrm{j}} \nabla^{2} \sigma+\frac{1}{r^{\prime}} \frac{[\psi, \sigma]}{\left[r^{\prime}, \theta\right]}+\frac{K_{v}}{\rho j}\left(\zeta^{\prime}-2 \sigma\right)
\end{aligned}
$$


$\frac{\partial T}{\partial \tau}=\frac{k}{\rho c_{v}} \nabla^{2} \mathrm{~T}+\frac{1}{r^{\prime}} \frac{[\psi, T]}{\left[r^{\prime}, \theta\right]}$

where

$$
\begin{aligned}
& \nabla^{2}=\frac{\partial^{2}}{\partial r^{\prime 2}}+\frac{1}{r^{\prime}} \frac{\partial}{\partial r^{\prime}}+\frac{1}{r^{\prime 2}} \frac{\partial^{2}}{\partial \theta^{2}} \quad \text { and } \\
& \frac{[\psi, s]}{\left[r^{\prime}, \theta\right]} \equiv \frac{\partial \psi}{\partial r^{\prime}} \frac{\partial s}{\partial \theta}-\frac{\partial s}{\partial r^{\prime}} \frac{\partial \psi}{\partial \theta} ; s \equiv\left[\zeta^{\prime}, \sigma, T\right]
\end{aligned}
$$

$\mathrm{K}_{\mathrm{v}}, \mathrm{j}$ and $\gamma$ are the vortex viscosity, micro-inertia density and spin-gradient viscosity. $\tau$ is the time, $\rho$ is the density, $k$ is the thermal' conductivity and $c_{v}$ is the specific heat. $\zeta^{\prime}$ is the vorticity, $\psi^{\prime}$ is the stream function, $T$ is the temperature and $\sigma$ is the component of microrotation vector whose direction of rotation is in the $\mathbf{r}^{\prime}-\theta$ plane.

The boundary conditions to be satisfied are the noslip, impermeability and isothermal conditions on the cylinder surface together with the free stream conditions very far away from the cylinder surface. These conditions can be expressed as

at $r^{\prime}=c$

$\psi^{\prime}=\frac{\partial \psi^{\prime}}{\partial \theta}=0, \frac{\partial \psi^{\prime}}{\partial r^{\prime}}=0$, and $\mathrm{T}=\mathrm{T}_{s}$

as, $r^{\prime} \rightarrow \infty \frac{\partial \psi^{\prime}}{\partial \theta} \rightarrow r^{\prime} U \cos \theta, \frac{\partial \psi^{\prime}}{\partial r^{\prime}} \rightarrow-U \sin \theta$,

$\zeta^{\prime} \rightarrow 0$ and $T \rightarrow T_{\infty}$

For micro-rotation, $\sigma$, the boundary conditions are assumed as

$$
\begin{array}{ll}
\text { At } r^{\prime}=c & \sigma=\zeta / 2 \\
\text { as } r^{\prime} \rightarrow \infty & \sigma \rightarrow 0
\end{array}
$$

The dimensionless forms of the above equations are obtained by introducing the following dimensionless variables

$r=\frac{r^{\prime}}{c}, \mathrm{t}=\frac{U t^{\prime}}{c}, \psi=\frac{\psi^{\prime}}{U c}, \zeta=-\zeta^{\prime} \frac{c}{U}, \mathrm{M}=\sigma \frac{\mathrm{c}}{\mathrm{U}}$,

$D=\frac{K_{v}}{\mu}, B=\frac{j}{c^{2}}, \quad \lambda=\frac{\gamma}{c^{2} \mu}$ and $\phi=\frac{T-T_{\infty}}{T_{s}-T_{\infty}}$

Using the above variables in equations (1)-(4) and using the modified polar coordinates $(\xi, \theta, \xi=\ln r)$ results in

$e^{2 \xi} \frac{\partial \zeta}{\partial t}=\frac{2(1+D)}{\operatorname{Re}} \nabla^{2} \zeta+\frac{[\psi, \zeta]}{[\xi, \theta]}+\frac{2 D}{\operatorname{Re}} \nabla^{2} \mathrm{M}$

$e^{2 \xi} \zeta=\nabla^{2} \psi$

$$
\begin{aligned}
& e^{2 \xi} \frac{\partial M}{\partial t}=\frac{2 \lambda}{\operatorname{Re} \cdot B} \nabla^{2} M+\frac{[\psi, M]}{[\xi, \theta]}-\frac{2 D}{\operatorname{Re} \cdot B}(\zeta+2 M) e^{2 \xi} \\
& \mathrm{e}^{2 \xi} \frac{\partial \phi}{\partial t}=\frac{2}{\operatorname{Re} \cdot \operatorname{Pr}} \nabla^{2} \phi+\frac{[\psi, \phi]}{[\xi, \theta]}
\end{aligned}
$$

where, $\operatorname{Re}=\frac{2 \rho U c}{\mu}$ is the Reynolds number and $\operatorname{Pr}=\frac{\mu}{\rho \alpha}$ is the Prandtl number.

The boundary conditions (5) based on the new variables can be expressed as

at $\xi=0 ; \psi=\frac{\partial \psi}{\partial \theta}=\frac{\partial \psi}{\partial \xi}=0$,

$M=-\zeta / 2$ and $\phi=1$

as $\xi \rightarrow \infty$;

$$
\frac{\partial \psi}{\partial \theta} \rightarrow e^{-\xi} \cos \theta, \frac{\partial \psi}{\partial \xi} \rightarrow-e^{-\xi} \sin \theta,
$$$$
\zeta \rightarrow 0, \mathrm{M} \rightarrow 0 \text { and } \phi \rightarrow 0
$$

The micropolar fluid flow is assumed to start impulsively from rest. The fluid around the cylinder is assumed initially at the ambient temperature (i.e $\phi=0)$.

\section{THE METHOD OF SOLUTION}

The method of solution is based on integrating the governing equations of motion and energy in time to obtain the velocity and temperature fields. Using the Fourier Spectral method and following the works of Badr and Dennis [17] and Mahfouz and Badr [18] the dimensionless stream function $\psi$, vorticity $\zeta$, Microrotation $M$ and temperature $\phi$ are approximated as follows :

$$
\begin{aligned}
& \psi=\frac{1}{2} \mathrm{~F}_{\mathrm{o}}+\sum_{n=1}^{N}\left[f_{n} \sin (n \theta)+F_{n} \cos (n \theta)\right], \\
& \zeta=\frac{1}{2} \mathrm{G}_{\mathrm{o}}+\sum_{n=1}^{N}\left[g_{n} \sin (n \theta)+G_{n} \cos (n \theta)\right] \\
& M=\frac{1}{2} \mathrm{R}_{\mathrm{o}}+\sum_{n=1}^{N}\left[r_{n} \sin (n \theta)+R_{n} \cos (n \theta)\right] \\
& \phi=\frac{1}{2} \mathrm{H}_{\mathrm{o}}+\sum_{n=1}^{N}\left[h_{n} \sin (n \theta)+H_{n} \cos (n \theta)\right]
\end{aligned}
$$

where $F_{o}, f_{n}, F_{n}, G_{o}, g_{n}, G_{n}, R_{o}, r_{n}, R_{n}, H_{0}, h_{n}$, and $H_{n}$ are the Fourier coefficients and $N$ represents the number of terms considered in Fourier series. Substitution of Equations (11) in Equations (1)-(4) results in the following set of differential equations: 


$$
\begin{aligned}
& \frac{\partial^{2} F_{o}}{\partial \xi^{2}}=e^{2 \xi} G_{o} \\
& \frac{\partial^{2} f_{n}}{\partial \xi^{2}}-n^{2} f_{n}=e^{2 \xi} g_{n} \\
& \frac{\partial^{2} F_{n}}{\partial \xi^{2}}-n^{2} F_{n}=e^{2 \xi} G_{n} \\
& e^{2 \xi} \frac{\partial G_{o}}{\partial t}=\frac{2(1+D)}{\operatorname{Re}} \frac{\partial^{2} G_{o}}{\partial \xi^{2}}+S_{o} \\
& e^{2 \xi} \frac{\partial g_{n}}{\partial}=\frac{2(1+D)}{\operatorname{Re}}\left(\frac{\partial^{2} g_{n}}{\partial \xi^{2}}-n^{2} g_{n}\right)+S_{n 1} \\
& e^{2 \xi} \frac{\partial G_{n}}{\partial}=\frac{2(1+D)}{\operatorname{Re}}\left(\frac{\partial^{2} G_{n}}{\partial \xi^{2}}-n^{2} G_{n}\right)+S_{n 2} \\
& e^{2 \xi} \frac{\partial R_{o}}{\partial t}=\frac{2 \lambda}{\operatorname{Re} \cdot B} \frac{\partial^{2} R_{o}}{\partial \xi^{2}}+K_{o} \\
& e^{2 \xi} \frac{\partial r_{n}}{\partial t}=\frac{2 \lambda}{\operatorname{Re} \cdot B}\left(\frac{\partial^{2} r_{n}}{\partial \xi^{2}}-n^{2} r_{n}\right)+K_{n 1} \\
& e^{2 \xi} \frac{\partial R_{n}}{\partial t}=\frac{2 \lambda}{\operatorname{Re} \cdot B}\left(\frac{\partial^{2} R_{n}}{\partial \xi^{2}}-n^{2} R_{n}\right)+K_{n 2} \\
& \mathrm{e}^{2 \xi} \frac{\partial \mathrm{H}_{0}}{\partial \mathrm{t}}=\frac{2}{\operatorname{Re} \operatorname{Pr}} \frac{\partial^{2} \mathrm{H}_{\mathrm{o}}}{\partial \xi^{2}}+\mathrm{Z}_{\mathrm{o}} \\
& e^{2 \xi} \frac{\partial h_{n}}{\partial t}=\frac{2}{\operatorname{Re} \operatorname{Pr}}\left(\frac{\partial^{2} h_{n}}{\partial \xi^{2}}-n^{2} h_{n}\right)+Z_{n 1} \\
& e^{2 \xi} \frac{\partial H_{n}}{\partial t}=\frac{2}{\operatorname{Re} \operatorname{Pr}}\left(\frac{\partial^{2} H_{n}}{\partial \xi^{2}}-n^{2} H_{n}\right)+Z_{n 2}
\end{aligned}
$$

where $S_{0}, S_{n 1}, S_{n 2}, K_{o}, K_{n 1}, K_{n 2}, Z_{o}, Z_{n 1}$ and $Z_{n 2}$ are all easily identifiable functions of $\xi$ and $t$. Equations (12a)-(15c) define a set of $(8 \mathrm{~N}+4)$ differential equations that have to be solved at every time step to get the flow and thermal fields. The boundary conditions for all functions present in Equations (12-15) are deduced from (10) and can be expressed as

at $\xi=0$

$\mathrm{F}_{\mathrm{o}}=\mathrm{F}_{\mathrm{n}}=\mathrm{f}_{\mathrm{n}}=\partial \mathrm{F}_{\mathrm{n}} / \partial \xi=\partial \mathrm{f}_{\mathrm{n}} / \partial \xi=\partial \mathrm{F}_{\mathrm{0}} / \partial \xi=0, \mathrm{R}_{\mathrm{o}}=-0.5 \mathrm{G}_{0}$,

$\mathrm{R}_{n}=-0.5 \mathrm{G}_{n}, \mathrm{r}_{n}=-0.5 \mathrm{~g}_{n}, \mathrm{H}_{0}=2$, and $\mathrm{H}_{\mathrm{n}}=\mathrm{h}_{n}=0$

and as $\xi \rightarrow \infty$

$\mathrm{F}_{\mathrm{o}}, \partial \mathrm{F}_{\mathrm{o}} / \partial \xi, \mathrm{F}_{\mathrm{n}}, \partial \mathrm{F}_{\mathrm{n}} / \partial \xi \rightarrow 0, \mathrm{f}_{\mathrm{n}} \rightarrow \delta_{1, \mathrm{n}}, \mathrm{e}^{-\xi} \partial \mathrm{f}_{\mathrm{n}} / \partial \xi \rightarrow$ $\delta_{1, n}, G_{0}, G_{n}, g_{n}, R_{0}, R_{n}, r_{n} \rightarrow 0$

$$
\text { and } \mathrm{H}_{\mathrm{o}}, \mathrm{H}_{\mathrm{n}}, \mathrm{h}_{\mathrm{n}} \rightarrow 0
$$

Integrating both sides of Equation (12a) with respect to $\xi$ between $\xi=0$ and $\xi=\infty$ and using the boundary conditions in Equation (16) gives the following integral condition:

$$
\int^{\infty} e^{2 \xi} G_{o} d \xi=0
$$

Similarly, integrating both sides of Equations (12b) and (12c) and making use of the boundary conditions (16), one can obtain the following integral conditions

$$
\begin{aligned}
& \int^{\infty} e^{(2-n) \xi} g_{n} d \xi=2 \delta_{1, n} \\
& \int^{\infty} e^{(2-n) \xi} G_{n} d \xi=0
\end{aligned}
$$

where $\delta_{1, \mathrm{n}}=1$ if $n=1$ and $\delta_{l, \mathrm{n}}=0$ if $\mathrm{n} \neq 1$

The above integral conditions are used at every time step to calculate the values of the functions $G_{o}, g_{n}$ and $G_{n}$ on the cylinder surface $(\xi=0)$. These functions are then used to compute accurately the vorticity distribution on the cylinder surface. The first condition (17a) is essential to ensure the periodicity of the pressure on the surface. The rest of the details of the method of solution and the numerical treatment is similar to that used in refs.[17-19] and will not be repeated here for the sake of brevity.

\section{RESULTS AND DISCUSSION}

The governing equations along with boundary conditions are solved in order to give the details of both flow and thermal fields. The main controlling parameters beside the classical ones ( Re and Pr) are the dimensionless micropolar material parameters. These are vortex viscosity $D$, spin viscosity $\lambda$ and microinertia density B. The close scrutiny of Eq.(6) and Eq. (8) shows that the vortex viscosity represents a direct link between the flow field and microrotation field. The other two dimensionless material parameters (spin viscosity and microinertia density B) appear only in microrotation, Eq. 8 and so they indirectely affect flow and thermal fields. For this reason and for the sake of brevity this study focuses only on the effect of the vortex viscosity on flow and thermal fields. The vortex viscosity is varied in the range from 0 to 5 while the spin viscosity and microinertia density are assigned value of 1 . The Reynolds number is varied up to 200 while Prandtl number is fixed at 0.7 .

\section{Wake flow, vortex shedding and lift coefficient}

With the start of computation, the flow commences impulsively over the cylinder surface. As the time goes the flow separates from the upper and lower surfaces of the cylinder, forming two symmetric vortices behind the cylinder. For Reynolds numbers less than a certain value ( about 40 for Newtonian fluids ), the flow over the cylinder remains stable and keeps horizontally symmetric with respect to the cylinder axis. The two generated 
symmetric vortices keep attached behind the cylinder and the wake length continues to grow with time till the quasi-steady state condition is almost reached at late times. The wake length is the length (divided by cylinder radius) of the separated wake measured along the line $\theta=0$ from the rearmost point of the cylinder to the end of re-circulating region. The calculations carried out for different cases for the symmetric flow (without vortex shedding) show that the wake length at a certain Reynolds number is greatly affected by the vortex viscosity. The results showed that as the vortex viscosity increases the wake length clearly decreases. The effect of increasing vortex viscosity seems similar to the effect of decreasing Reynolds number, that is to say that the flow becomes more viscous as the vortex viscosity increases.

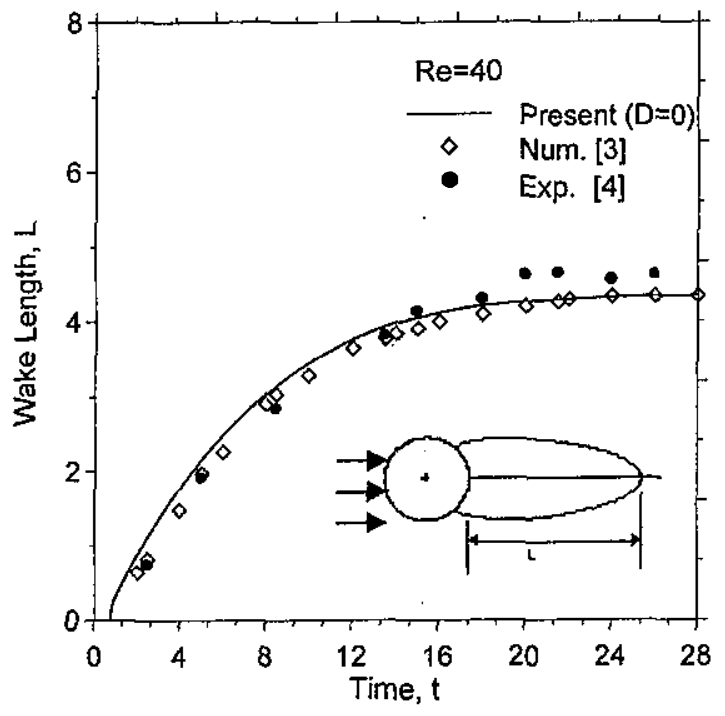

Fig. 1 Time development of wake length for the case $\operatorname{Re}=40, D=0$ and comparison with refs. [3] and [4]

Fig. 1 shows the time development of the wake length for the case of symmetric flow of a Newtonian fluid $(D=0)$ at low Reynolds number of 40 . Shown also in the same figure are the numerical results of Collins and Dennis [3] and experimental results of Honji and Tanida [4]. The figure shows very good agreement with numerical results of Collins and Dennis [3] at both initial and late times, while the comparison with the experimental results of Honji and Tanida [4] is very good up to time $t=18$ and reasonable for $t>18$. Further rigorous testing of the method of solution can be found in the works of Mahfouz and Badr $[18,19]$

As the Reynolds number exceeds a certain value the flow in the cylinder wake becomes unstable and vortices are shed alternately and periodically from the upper and lower sides of the cylinder, forming the well known Karman vortex street. In the present numerical treatment, in order to excite the Karman vortex street, the flow is intentionally perturbed by rotationally oscillating the cylinder for only one complete cycle. The amplitude and duration of the cycle were selected in order to trigger the vortex shedding as rapidly as possible without causing any long-time effects. The frequency of vortex shedding, $f_{0}$, is computed from Fourier frequency analysis (FFT) of the time record of lift coefficient.

Table 1 Strouhal number and time averaged Nusselt number at different $\mathrm{Re}$ and $\mathrm{D}$

\begin{tabular}{|c|c|c|c|c|c|c|c|}
\hline $\mathrm{Re}$ & D & $S_{o}$ & $\overline{\overline{N U}}$ & $\operatorname{Re}$ & $\mathrm{D}$ & $\mathrm{S}_{\mathrm{o}}$ & $\overline{\overline{N U}}$ \\
\hline \multirow{4}{*}{20} & 0.0 & $-\cdots$ & 2.52 & \multirow{4}{*}{100} & 0.0 & .160 & 5.32 \\
\hline & 0.1 & $m-$ & 2.51 & & 0.5 & .156 & 5.23 \\
\hline & 0.5 & 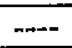 & 2.45 & & 1.0 & .120 & 4.41 \\
\hline & 2.0 & -- & 2.38 & & 5.0 & $m$ & 4.32 \\
\hline \multirow{4}{*}{50} & 0.0 & .134 & 3.79 & \multirow{4}{*}{200} & 0.0 & .180 & 6.98 \\
\hline & 0.1 & .130 & 3.75 & & 0.5 & .170 & 6.79 \\
\hline & 0.5 & 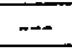 & 3.37 & & 1.0 & .165 & 6.69 \\
\hline & 2.0 & $\therefore$ & 3.31 & & 2.0 & .145 & 6.49 \\
\hline
\end{tabular}

The dimensionless frequency of vortex shedding is known as Strouhal number $\left(\mathrm{S}_{0}=2 . f_{0} \mathrm{c} / \mathrm{U}\right)$. The Strouhal number depends not only on the Reynolds number (as in case of Newtonian fluids ) but also on the material parameters of micropolar fluid. The numerical results for Strouhal number at various values of Re and D are displayed in Table 1, together with the results of time averaged mean Nusselt number $\overline{\overline{N u}}$. The results of $\overline{N u}$ will be explained later. The table shows that the Strouhal number for the cases of Newtonian fluids $(D=0)$ compares very well with the corresponding ones in refs. $[18,20$, 21]. The table also shows clearly that as the vortex viscosity increases the frequency of vortex shedding decreases. The void (dashed) entries in the table mean that the flow will be symmetric (i.e no vortex shedding) at very large time.

In order to figure out further the effect of yortex viscosity on vortex shedding process let us examine the lift coefficient. The lift coefficient is defined as $C_{L}=L F / c \rho U^{2}$ where LF is the lift forces exerted on a unit length of the cylinder. In terms of Fourier coefficients the lift coefficients can be expressed as

$C_{L}=-\frac{2 \pi}{\operatorname{Re}}\left\{(1+D)^{*}\left[G_{1}-\frac{\partial G_{1}}{\partial \xi}\right]+D\left[R_{1}-\frac{\partial R_{1}}{\partial \xi}\right]\right\}_{x}$

For the case of symmetric flow over the cylinder the lift force vanishes as a result of equally opposing (pressure and viscous) forces acting on the upper and lower sides of the cylinder. With the development of periodic shedding of vortices the flow in the cylinder wake becomes unsteady and asymmetric which leads to asymmetric distributions of pressure and viscous 
forces. These asymmetric distributions lead to periodic time variation in lift coefficient. The frequency of lift coefficient oscillation is the same as the vortex shedding frequency. This is mainly due to the nature of vortex shedding mechanism in which vortices of opposite circulation shed alternately from upper and lower sides of the cylinder surface. The period of $C_{L}$ oscillation is equal to the time taken to detach two consecutive vortices, one from the upper side and the other from the lower side of the cylinder. The variation of $C_{L}$ is positive for one vortex and negative for the other due to opposite circulation.

Fig. 2 shows the time variation of the lift coefficient for the case of $\operatorname{Re}=50$ and $D=0,0.1,0.5$ and $D=2$. The figure shows clearly that as vortex viscosity increases the amplitude of lift forces decreases. The lift coefficients for the cases $D=0.0$ and $D=0.1$ oscillate with almost constant amplitude which reflects the persistent nature of vortex shedding for these two cases. For higher values of $D=0.5$ and $D=2$ the amplitude of lift coefficient is no longer constant but rather is continuously decaying with time, expectedly reaching zero after a long time. The rate of $\mathrm{C}_{\mathrm{L}}$ decaying as shown in the figure for the last two cases is much faster for the bigger values of $D$. The full diminishing of lift forces at large times indicates that the flow will eventually be symmetric. So, it can be inferred from Table 1 and Fig. 2 that the increase of vortex viscosity beyond a certain value may lead to a decrease in the flow response to the perturbation with tendency of the flow to be symmetric (i. e. no vortex shedding ) at large times. In this study, however, no attempts - were made to find the exact value of $\mathrm{D}$ (at certain $\mathrm{Re}$ ) at which the flow at large time becomes steady symmetric. This finding is important as far as the vortex shedding and separation control is concerned. The closer the behavior of the fluid to the micropolar fluid the weaker the possibility of vortex shedding.

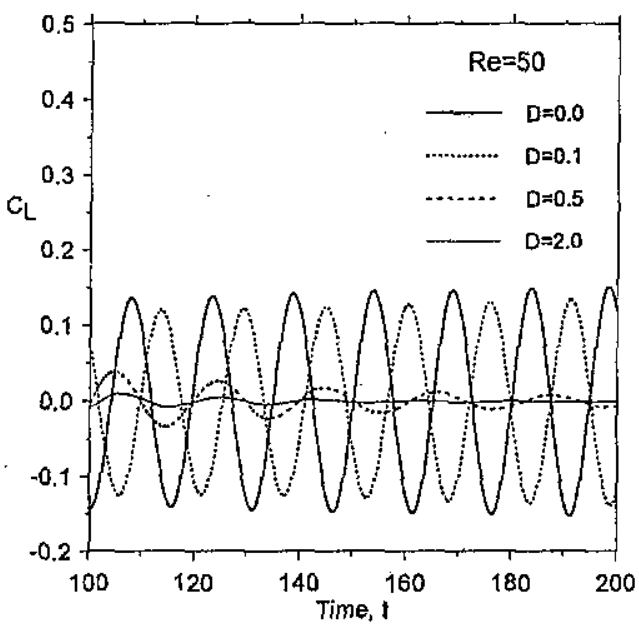

Fig. 2 Time variation of lift coefficient for the case of $\operatorname{Re}=50$ and $D=0,0.1,0.5$, and 2 .
The variation of microrotation $M$ which represents micro-elements rotation as well as the variation of vorticity $\zeta$ which represents the mean flow rotation in the cylinder wake along the rear axis $(\theta=0)$ is shown in Fig. 3 for the case of $R e=50, D=0.1$ and at $t=200$. The figure shows that both $M$ and $\zeta$ fluctuates along the rear axis due to vortex shedding with decaying amplitude as a result of viscous diffusion. Also the figure clearly shows that almost at all points $M$ is one-half that of $\zeta$ which means that micro-elements are rotating at the mean flow local rotating velocity.

\section{Heat transfer results}

Immediately following the impulsive flow motion over the constant temperature cylinder surface, the heat transfer rate gets very high values as a result of heat conduction through very thin thermal layer. In the early time stages, the fast growing of thermal boundary layer resulted in drastic decrease in heat transfer in a relatively short time. After that short time the thermal layer is almost developed with heat transfer tending to assume almost steady value (in case if the flow is still not perturbed ). Once the flow is perturbed the vortex shedding process develops and causes flow unsteadiness in the vicinity of the cylinder. Such unsteadiness cause an enhancement in mean heat convection in comparison with steady symmetric situation. In order to quantify the heat transfer results let us define the surface local and mean Nusselt numbers:

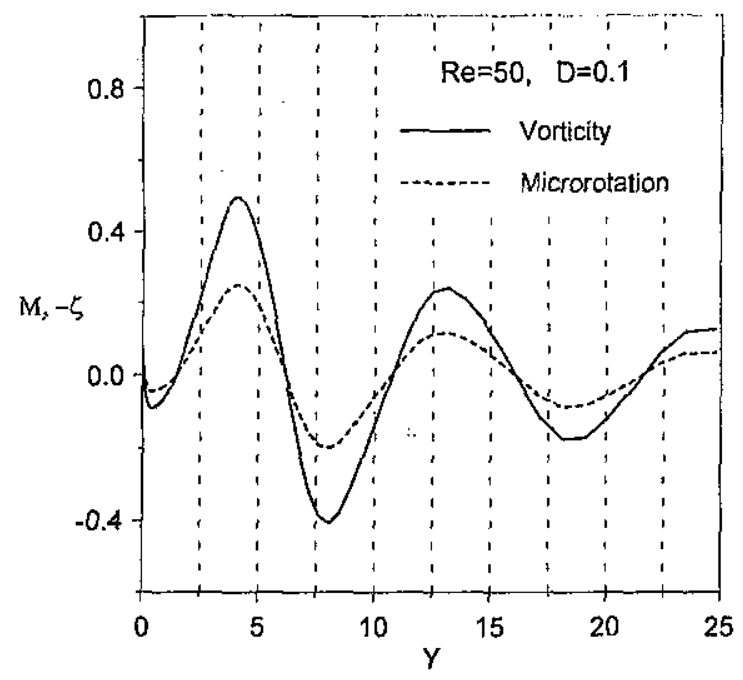

Fig. 3 Variation of microrotation $M$, and vorticity, $-\zeta$ in the cylinder wake along the rear axis $(\theta=0)$,

$N u=\frac{2 q^{\prime} \mathrm{c}}{k\left(T_{s}-T_{\infty}\right)}$ and $\overline{N u}=\frac{1}{2 \pi} \int_{0}^{2 \pi} N u \mathrm{~d} \theta$

$q^{\prime}$ is heat transfer per unit area given by $q^{\prime}=-\left.k \frac{\partial T}{\partial r^{\prime}}\right|_{r^{\prime}=c}$. From the above definitions and 
using Equation (11), one can deduce the following relation for local and mean Nusselt numbers

$$
N u=-2\left(\frac{\partial \phi}{\partial \xi}\right)_{\xi=0} ; \quad \overline{N u}=-\left(\frac{\partial H_{0}}{\partial \xi}\right)_{\xi=0}
$$

The time-averaged Nusselt number is obtained from

$$
\overline{\overline{N u}}=\frac{1}{t_{2}-t_{1}} \int_{t_{1}}^{t_{2}} \overline{N u} \mathrm{dt}
$$

where the time period between $t_{1}$ and $t_{2}$ covers a good number of cycles of vortex shedding.

Fig. 4 shows the steady mean Nusselt number distribution for the case of symmetric low Reynolds numbers $1<\operatorname{Re}<40$ Newtonian fluid flow $(D=0)$ at value of $\operatorname{Pr}=0.73$. Shown also in the same figure for the purpose of comparison are the experimental correlation due to Hatton et al. [22] and Knudsen and Katz [23]. The correction factors for temperature in both works have been taken as one. The comparison with these two correlation is very good with maximum difference less than $10 \%$ in case of comparison with Hatton et al. [22] and less than 7\% in case of comparison with Knudsen and Katz [23].

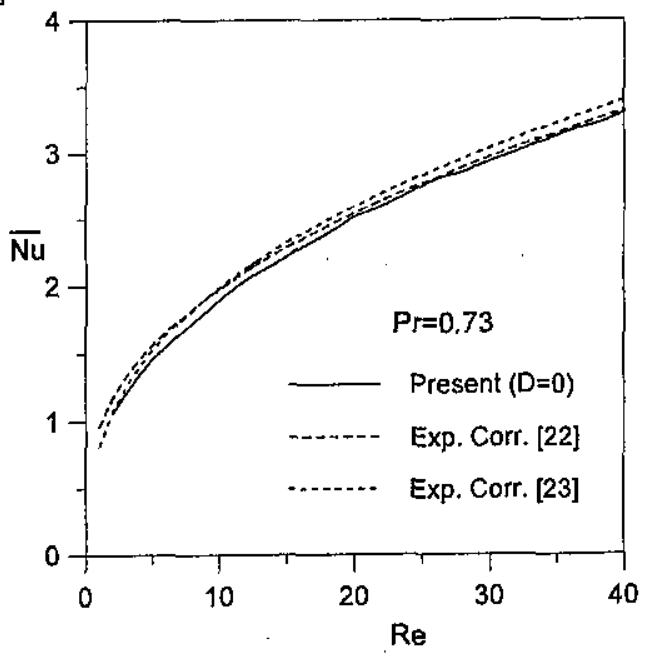

Fig. 4 Variation of $\overline{N u}$ with $\operatorname{Re}$ and comparison with the correlation of [22] and [23]

Fig. 5 shows the late time variation of surface mean Nusselt number, $\overrightarrow{\mathrm{Nu}}$ for the unsteady flow for the case of $\operatorname{Re}=200, \operatorname{Pr}=0.7$ and $D=0,0.5,1,5$. The figure shows that $\overline{N u}$ fluctuates around a mean value and the amplitude of these fluctuations are more pronounced for smaller values of $D(D=0$ and $D=0.5)$. While at bigger values of $D$ the amplitude of fluctuation is getting smaller. The frequency of fluctuation is twice the frequency of vortex shedding. It can be also observed from the figure that the timeaveraged value of $\overline{N u}$ is getting markedly smaller as $\mathrm{D}$ increases. The numerical results of time averaged Nusselt number, $\overline{\mathrm{Nu}}$ for the cases considered in this study are listed in Table 1. The table shows that as $\mathrm{D}$ increases $\overline{\overline{N u}}$ decreases. This finding is consistent with the previous results of Mahfouz [24-25] for natural convection from circular and elliptic cylinders placed in micropolar fluids and so can be considered as a general conclusion for heat convection (forced or natural) in micropolar fluids.

Shown in Fig. 6 are the local Nusselt number, $\mathrm{Nu}$ distributions for the unsteady flow for the case of $\mathrm{Re}=100, \mathrm{Pr}=0.7, \mathrm{D}=0,0.5,1,5$ and at late time $t=150$. The figure shows that $\mathrm{Nu}$ distributions for micropolar fluid are similar to that of Newtonian fluid $(D=0)$. The figure shows that for all distributions $\mathrm{Nu}$ gets its maximum value at the front stagnation point $(\theta=180)$ or in its vicinity. At the front stagnation point the thermal layer is minimum which results in maximum heat rate at that point. The $\mathrm{Nu}$ then decreases due to thermal boundary layer growth in the direction of flow on upper and lower sides of the cylinder till it gets its minimum value near the rear most point of the cylinder $(\theta=0)$. In the rear region and as a result of vortex shedding the heat transfer rate and so $\mathrm{Nu}$ assumes fluctuating and relatively higher values as compared with steady cases (not shown here).

The figure also shows a clear reduction in Nusselt number at almost all points of the surface as vortex viscosity increases. The reduction in Nu at almost all points at the surface means the reduction of mean values of $\overrightarrow{\mathrm{Nu}}$ as the vortex viscosity increases. The decrease of local values of $\mathrm{Nu}$ as vortex viscosity increases may be explained in the light of surface vorticity distributions shown in Fig. 7 for the same cases and the same time. Higher values of surface vorticity at the same location ( same $\theta$ ) means higher flow velocity gradient (i.e higher convection ) near the surface which results in higher heat transfer (i.e higher $\mathrm{Nu}$ ).

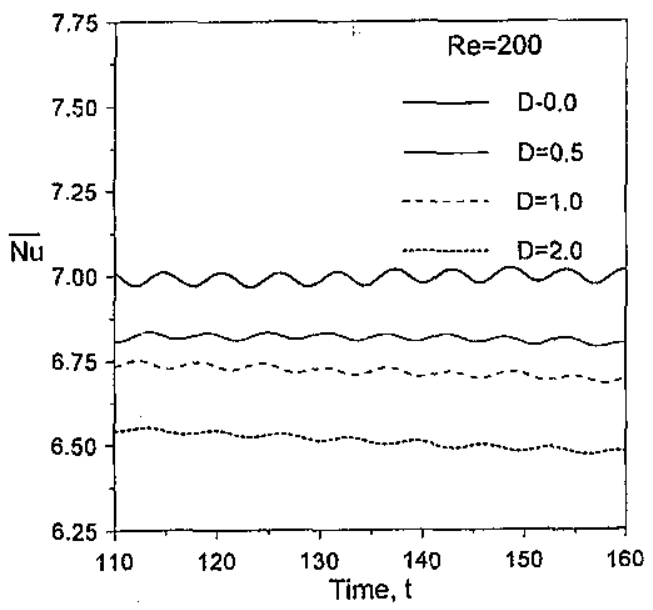

Fig. 5 Time variation of mean Nusselt number at $\operatorname{Re}=200, \operatorname{Pr}=0.7$ and different values of $\mathrm{D}$. 


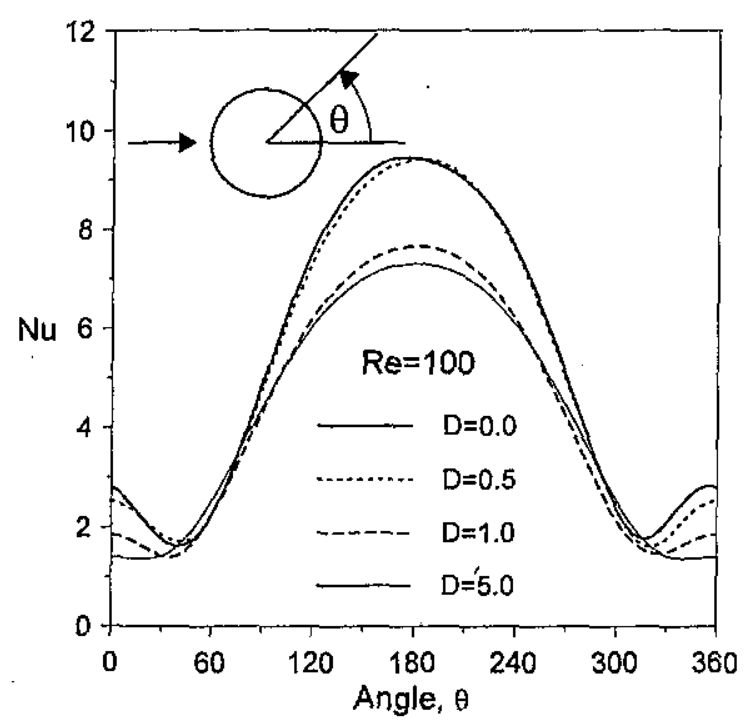

Fig. 6 Distribution of Surface local $\mathrm{Nu}$ at $\mathrm{Re}=100$, $P r=0.7, t=150$ and at different values of $D$

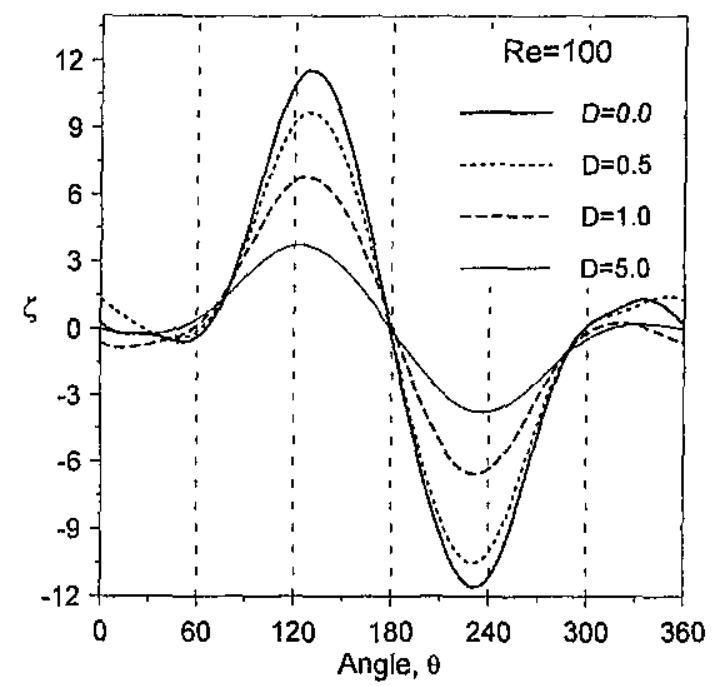

Fig. 7 Distribution of surface vorticity at $\mathrm{Re}=100$, $\mathrm{Pr}=0.7, \mathrm{t}=150$ and at different values of $\mathrm{D}$

With the increase of the vortex viscosity the flow becomes more viscous and the low velocity gradient near wall results in low convection currents and so low local heat transfer as shown in Fig. 6. The maximum heat transfer rate at front stagnation point is not controlled by flow velocity gradient, which is zero at that point, but is rather controlled by the thickness of thermal layer which is minimum at that point.

Fig. 8 shows the dimensionless temperature distribution in the wake of the cylinder along the rear axis $(\theta=0)$ at time of $t=150$ and for the case of $\mathrm{Re}=100, \mathrm{Pr}_{\mathrm{r}}=0.7$ and $\mathrm{D}=0,0.5,1$, 5. The figure shows that the temperature decreases in the wake for all cases, expectedly reaching the value of unheated fluid very far away from the cylinder $(\phi=0)$. The figure shows clearly that for the cases of $D=0,0.5$ and 1 the temperature fluctuates during its decreasing while for the case of $D=5$ it monotonically decreases. These distributions of temperature again confirms the results shown in Table 1 that the wake flow is characterized by vortex shedding for the cases of $D=$ $0,0.5,1$ while for the case of $D=5$ the symmetric wake flow without vortex shedding is reached at that time. The temperature distributions as shown in the figure reveal that as the value of $D$ increases the temperature gradient at the surface decreases, declaring a smaller local heat transfer rates and, accordingly, as shown in Fig. 6, smaller $\mathrm{Nu}$ as D increases.

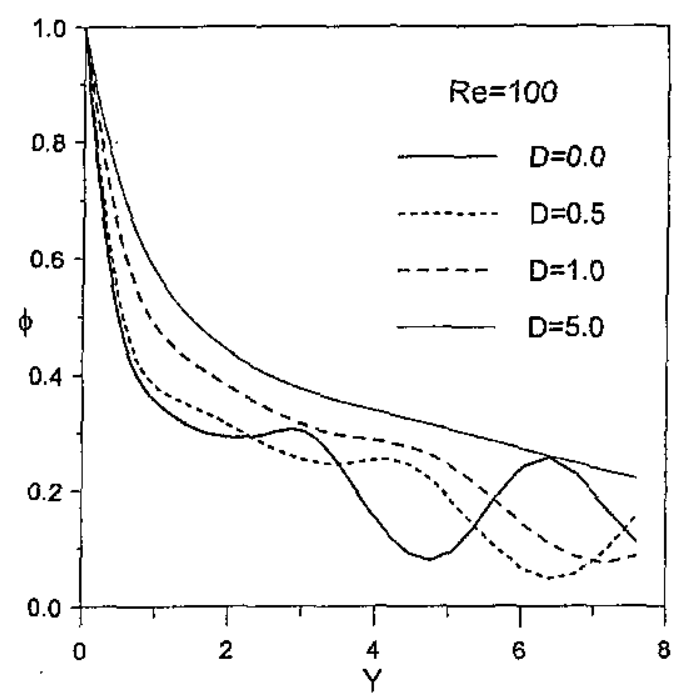

Fig. 8 Temperature distribution in the wake of the cylinder along line $\theta=0$

Fig. 9 shows the thermal field, represented by contour plots of isotherm patterns for the case of $\operatorname{Re}=50$ and at time $t=200$ and at four values of $D$ namely, $D=0.0,0.1,0.5$, and $D=2$. The flow is perturbed in the four cases at $t=30$. The figure shows that for the value of $D=0.0$ and 0.1 (Fig. 9a and Fig. 9b) the isotherms reflect the asymmetry of the flow which is persistently characterized by vortex shedding which is clear in time variation of lift coefficient (see Fig. 2). For larger value of vortex viscosity, $\mathrm{D}=0.5$ ( Fig. 9c) the isotherms are about to be symmetric while for much larger value of $D=2$ ( Fig. 9d) the perturbation effect is already damped and the flow and thermal fields became fully symmetric. The dashed contour line in Fig. 9 represents the isotherm contour of $\phi=0.1$ which can be considered as a qualitative indicator of the thickness of the thermal layer. The figure clearly shows that the dashed contour encloses wider loop around the cylinder as D increases which indicates that the thermal layer around the cylinder gets thicker as the value of $\mathrm{D}$ increases. A thicker thermal layer means smaller temperature gradient near the cylinder surface and in turn smaller heat transfer rates. 

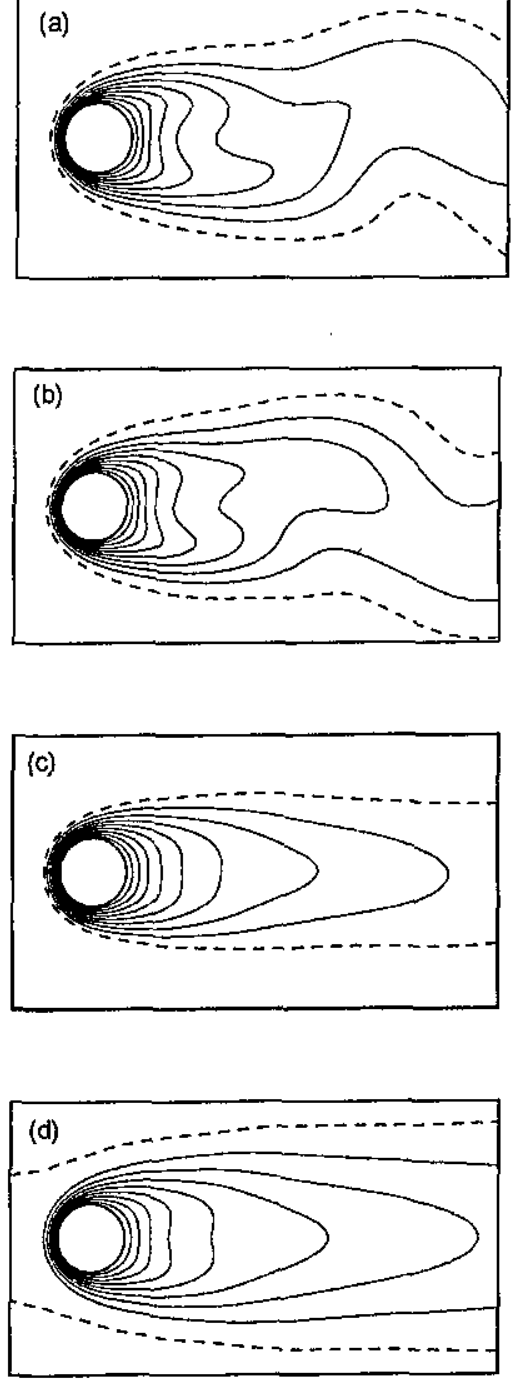

Fig. 9 Isotherm patterns for the case of $R e=50$, $t=200$, a) $D=0.0$, b) $D=0.1$, c) $D=0.5$ and d) $D=2$

\section{CONCLUSION}

This paper investigated forced convection and hydrodynamics forces associated with the flow of micropolar fluids over a horizontal circular cylinder. The effect of Reynolds number and material parameters of micropolar fluid are considered in this study. The Reynolds number is considered up to 200 . The vortex viscosity is the only material parameter considered in this study and is selected in the range from 0 to 5 . The results have shown a reduction in mean heat transfer rate as the vortex viscosity increases. Similarly, the natural vortex shedding frequency decreases as the vortex viscosity increases. The amplitude of oscillating lift force also experiences a clear reduction as the vortex viscosity increases.

\section{REFERENCES}

[1] Eckert, E.R.G. and Soehngen E., 1952 , "Distribution of heat transfer coefficient around circular cylinders in cross flow at Reynolds numbers from 20 to $500 ", \mathrm{~J}$, Heat Transfer 74 , $343-347$.

[2] Dennis, S. C. R., Hudson, J. D. and Smith, N., 1968 , "Steady laminar forced convection from a circular cylinder at low Reynolds numbers", Phys. Fluids 11 (5), $933-940$.

[3] Collins, W. M. and Dennis, S. C. R, 1973, "Flow past an impulsively started circular cylinder", J. Fluid Mechanics, 60(1), pp. 105127.

[4] Honji, H.and Taneda, S.,1969, "Unsteady flow past a circular cylinder", J. Phys. Soc. Japan, 27, pp. 1688-1698.

[5] Karniadakis, G. E., 1988, "Numerical simulation of forced convection heat transfer from a cylinder in cross flow", Int. J. Heat Mass Transfer 31 (1), $407-418$.

[6] Chun, W. and Boehm, R. F., 1989, "Calculation of forced flow and heat transfer around a cylinder in cross flow", Numer.Heat Transfer $15,101-122$.

[7] Eringen A. C., 1964, "Simple micropolars", Int. J. Eng. Sci. 2: 205-217

[8] Eringen A. C., 1972, "Theory of thermomicrofluids", J. Math. Anal. Appl. 38: 480-496

[9] Ariman T, Turk M. A. and Sylvester N. D., 1973, "Microcontinuum fluid mechanics, a review", Int. J. Eng. Sci. 11, 905-930

[10] Gorla, R. S. R, 1984, "Heat transfer characteristics of a micropolar boundary layer in a crossflow over a non-isothermal circular cylinder", Int. J. of Eng. Sci., Vol. 22(1), pp. $47-$ 55

[11] Hassanien, I. A, Mansou,r M .A. and Gorla, R. S. R., 1994, "Combined convection on a vertical slender cylinder in a micropolar fluid", Waerme und Stoffuebertragung 29, 355-359

[12] Gorla, R. S. R, 1995, "Axisymmetric thermal boundary layer of a micropolar fluid on a cylinder", Int. J. Eng. Sci. 23, 401-407

[13] Mohammedien, A. A., Gorla, R. S.R. and Hassanien, I. A., 1996, "Mixed convection in an axisymmetric stagnation flow of micropolar fluid on a vertical cylinder", Acta Mecanica. 114, 139149

[14] Hassanien, I, A. and Salama, A. A. 1997, "Flow and heat transfer of a micropolar fluid in an 
axisymmetric stagnation flow on a cylinder", Energy Cmuers. Mgmr Vol. 38, No. 3, pp. 301310

[15] Gorla, R.S.R. and Takhar, H. S.,1991, "Unsteady mixed convection boundary layer flow of a micropolar fluid near the lower stagnation point on a cylinder", Int. J. Eng. Fluid Mech. 4 (3), 337

[16] Mansour, M A., El-Hakiem, M A and El Kabeir, $S \mathrm{M}, 2000$, "Heat and mass transfer in magnetohydrodynamic flow of micropolar fluid on a circular cylinder with uniform heat and mass flux" ,Journal of Magnetism and Magnetic Materials 220, 259-270

[17] Badr, H. M. and Dennis, S. C. R, 1985, "Timedependent viscous flow past an impulsively started rotating and translating circular cylinder", J. Fluid Mech. 158, pp. 447-488

[18] Mahfouz, F. M. and Badr, H. M.,2000, "Flow structure in the wake of a rotationally oscillating cylinder", ASME J. of Fluids Engineering, 122, pp. 290-301.

[19] Mahfouz, F. M. and Badr, H. M., 2000, "Forced convection from a rotationally oscillating cylinder placed in a uniform stream, Int. J. Heat and Mass Transfer, 43(17), pp. 3093-3104

[20] Roshko, A.,1954, "On the development of turbulent wakes from vortex streets, NACA Rep., 1191.

[21] Jordon, S. K. and Fromm, J. E., 1972, "Oscillatory drag, lift and torque on a circular cylinder in a uniform flow", Physics of Fluids, 15(3), pp. 371-376.

[22] Hatton, A. P., James, D. D. and Swire, H. W., 1971, Combined forced and natural convection with low speed air flow over horizontal cylinders, J. Fluid Mechanics, 42, pp. 17-31.

[23] Knudsen, J.D., and Katz, D. L., 1958, Fluid dynamics and heat transfer, McGrow-Hill, New York,

[24] Mahfouz, F. M., 2003, "Transient free convection from a horizontal cylinder placed in a micropolar fluid", Heat and Mass Transfer, 39, pp. $455-462$.

[25] Mahfouz, F. M., 2004, "Natural convection from an elliptic tube with major axis horizontal and placed in micropolar fluid", Int. J. Heat and Mass Transfer, 47(6-7), pp. 1413-1422. 